\title{
Guaranteed Dynamic Localization using Constraints Propagation Techniques on Real Intervals
}

\author{
A. Gning and Ph. Bonnifait \\ Heudiasyc UMR 6599 \\ Université de Technologie de Compiègne. \\ BP 20529, 60205 Compiègne Cedex, France. \\ gningelh@hds.utc.fr
}

\begin{abstract}
In order to get continuous dynamic localization of a mobile robot, dead reckoning and absolute sensors are usually merged. The models used for this fusion are non linear and, therefore, classical tools (such as Kalman filter) cannot guarantee a maximum error estimation. In some applications, integrity is essential and the ability to guaranty the result is a crucial point. There are ensemblist approaches that are insensitive to non linearity. In this context, the random errors are only modeled by their maximum bound. This paper presents a new technique to merge the data of redundant sensors with a guaranteed result based on constraints propagation techniques on intervals. We have thus developed an approach for the fusion of the 4 ABS wheel encoders, a measure of the angle of the driving wheel and a differential GPS receiver. Experimental results show that the precision that one can obtain is very good with a guaranteed result. Moreover, constraints propagation techniques are well adapted to a real time implementation.
\end{abstract}

\section{INTRODUCTION}

Mobile robot localization is a well known problem. Usually, the localization process involves dead reckoning sensors (like odometry, gyros, etc.) and absolute sensors (like telemeters, goniometers, GPS, etc.). These two technologies are complementary and the fusion of the sensors measurements can bring more precision, availability and integrity. For example, GPS suffers from satellite masks occurring in forests, cities, tunnels, etc. In this case, a dead reckoning technique can still provide an estimation of the pose of the mobile robot. Moreover, by using continuously odometry in a fusion process, one can filter the GPS estimates.

Furthermore, the methods used in the fusion stage, like Kalman filtering often rely on a state space representation. Nevertheless, the state space representations considered in robotics are usually non linear (in the evolution and observation models). The classical solution is to linearize the equations around the previous estimated state and then apply a linear technique. This is the principle of the Extended Kalman Filter in Gaussian perturbations context. The main drawback of such an approach is that the convergence of the observer cannot be guaranteed. In practice, the observer can converge towards a local minimum which can be different of the real solution.

With the assumption that the model and measurement errors are bounded, completely different approaches can be studied. These ensemblist approaches (also known as "bounded error methods" or "set-membership methods") can be completely non linear. For example, in [2], the authors propose to use Sivia algorithm (Set Inversion by Interval Analysis) to find all the possible static 3D locations with an automatic theodolite using indistinguishable landmarks. If only one solution is obtained, then an EKF is applied. Ensemblist observers based on a predictor/estimator mechanism have also been proposed $[5,7$, 8]. The same technique has been used in [1] to localize a mobile robot with ultrasonic measurements. These approaches can reach a high precision with a guaranteed result, but they are not adapted to a real time implementation since they are very slow and since their computation time is not limited, because of the bisections of the state space used to find the result.

When there is a high redundancy of measurements and equations, propagation techniques on real intervals can be used. The key idea of such an approach is to find the consistence of all the data linked by the state space equations which provides what one calls "constraints". A remarkable property of these techniques is that they are very fast, compared to the bounded error predictor/estimator observers, and thus they are well adapted to a real time running.

The objective we consider in this work is to develop a real time bounded-error approach based on interval analysis, in order to guarantee the vehicle location on a terrestrial reference frame. We are not looking for a high precision but we want to achieve a high integrity in the localization process [12]. This is essential for driving assistance systems that use digital maps.

The sensors that we consider (the 4 ABS wheel encoders, a measure of the angle of the driving wheel and a differential GPS receiver) provide redundant data. Therefore, we propose to study in this paper how propagation techniques on real intervals can fuse all this information. 
The paper is organized as follows. Part II presents the fundamental notions of interval analysis, consistence and constraints propagation. The contractor we have used in the experiments is described. In section III, the equations of the discrete and non linear state space are provided. We have decomposed the fusion problem into two stages and we explain for both of them the ensemblist solution we have applied. Finally, section IV presents experimental results carried out with our experimental car.

\section{INTERVAL ANALYSIS AND CONSTRAINTS PROPAGATION}

In this section, we briefly present interval analysis and we explain the constraints propagation technique (also called consistence technique in the literature) used for the multisensor fusion process.

\section{A. Basic definitions}

A real interval, denoted $[x]$, is defined as a closed and connected subset of $I R$, and a box $[x]$ of $I R^{n}$ as a Cartesian product of $n$ intervals $\left([\mathrm{x}]=\bigotimes_{i=1 \ldots n}\left[\mathrm{x}_{\mathrm{i}}\right]\right)$.

The main idea of interval analysis is to provide efficient tools and different algorithms to guarantee with the least pessimism, the inclusion of all possible solutions of an ensemblist problem to a given box. All elementary arithmetic operations like $\{+,-, \times, /\}$ are extended to the ensemblist context [10]. Extensions are also defined for usual operations between sets of $I R^{n}$ like $\{\cap, \supset, \subset$, etc... $\}$.

The image of an interval by a function isn't necessarily an interval, so the inclusion function [11] has been developed to calculate efficiently an interval enclosing the image set.

For the fusion problem considered, we have chosen to use constraints propagation techniques, because of the great redundancy of data and equations.

\section{B. Constraints Satisfaction Problem}

Constraints Satisfaction Problems (CSP) were initially defined for discrete domains i.e. the values $x_{i}$ belongs to finite sets [4]. Later, CSP were extended to continuous domains (box of $I R^{n}$ in general) [5].

Consider a box $[x]$ of $I R^{n}$. Let consider $m$ relationships representing the constraints and linking the components $\left[x_{i}\right]$ of $[x]:$

$$
f_{i}\left(x_{1}, \ldots, x_{n}\right)=0, i=1 \ldots m
$$

Let $f$ be the Cartesian product of $f_{\mathrm{i}}$. Equation (1) can be rewritten in a vectorized form as $f(x)=0$

The problem of searching the minimal box of $[\mathrm{x}]$ satisfying all the constraints $f_{i}$ corresponds to a CSP denoted $\mathcal{H}$, which can be formulated as:

$$
\mathcal{H}:(f(x)=0 \mid x \in[x])
$$

The solution set of $\mathcal{H}$ is defined as

$$
S=\{x \in[x] \mid f(x)=0\}
$$

\section{Consistence}

A scalar $x_{i}$ belonging to the $\mathrm{i}^{\text {th }}$ component of $[x]$ is globally consistent with $\mathcal{H}$, if it is possible to find a vector $x$ within $S$ having it like $i^{\text {th }}$ coordinate.

$\exists x_{I} \in\left[x_{1}\right], \ldots, x_{i-1} \in\left[x_{i-1}\right], x_{i+1} \in\left[x_{i+1}\right], \ldots, x_{n} \in\left[x_{n}\right] / \mathrm{f}\left(x_{1}\right.$, $\left.\ldots x_{i-1}, x_{i}, x_{i+1}, \ldots, x_{n}\right)=0$

A scalar $x_{i}$ belonging to the $\mathrm{i}^{\text {th }}$ component of $[x]$ is locally consistent with $\mathcal{H}$ if for such constraint $f_{\mathrm{i}}$ (taken separately), it is possible to find a vector $x$ consistent with $f_{\mathrm{i}}$ having it like $i^{\text {th }}$ coordinate :

$\forall f_{i}, \exists x_{1} \in\left[x_{1}\right], \ldots, x_{i-1} \in\left[x_{i-1}\right], x_{i+1} \in\left[x_{i+1}\right], \ldots, x_{n} \in\left[x_{n}\right] /$ $f_{i}\left(x_{1}, \ldots, x_{n}\right)=0$

An interval $[x]$ is globally (respectively locally) consistent with $\mathcal{H}$ if $\forall x_{i} \in[x], x_{i}$ is globally (respectively locally) consistent with $\mathcal{H}$. Global consistence implies local consistence.
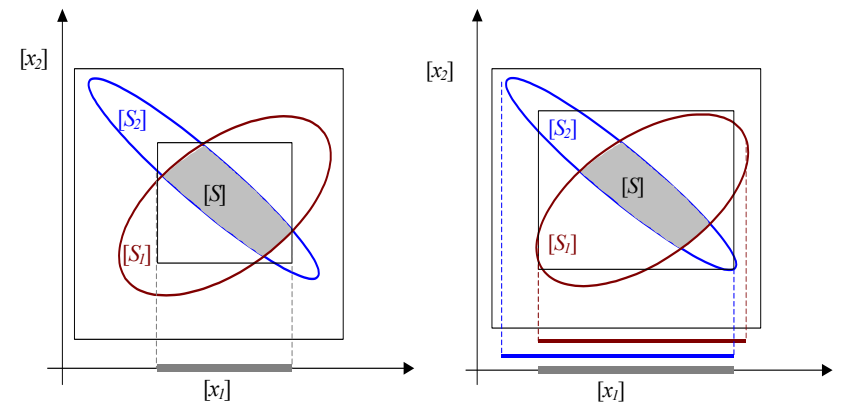

Fig. 1. Global (left) and local (right) consistence.
Figure 1 illustrates these notions: $S_{1}$ and $S_{2}$ are the solutions of two constraints considered separately, $\mathrm{S}$ is the solution of the CSP.

\section{Contractors}

To contract $\mathcal{H}$ means to substitute the box $[x]$ by a smaller box $\left[x^{\prime}\right] \subset[x]$ containing all the solutions of $\mathcal{H}$ i.e. $S \subset\left[x^{\prime}\right]$.

A contractor for $\mathcal{H}$ is defined as an operator used to contract $\mathcal{H}$.

There are different kinds of contractors [6]. In this paper, we use a forward backward propagation technique based on primitive constraints [4]. It is a locally consistent contractor called FBP in the following.

The principle is the following. Let consider the constraint $z=x \cdot \cos (y)$. At first, this constraint is decomposed into primitive constraints. A primitive constraint only involves an arithmetic operator or a usual function (cos, exp, etc.). 


$$
\left\{\begin{array}{c}
a=\cos (y) \\
z=x . a
\end{array}\right.
$$

where $a$ is an auxiliary variable initialized by $[\mathrm{a}]=[-\infty,+\infty]$. Let $[\cos ]$ and $\left[\cos ^{-1}\right]$ be inclusion functions for functions cos and $\cos ^{-1}$. The FBP works as follows.

$$
\begin{aligned}
& \text { \%forward propagation } \\
& {[a]=[a] \cap[\cos ]([y]) \quad \mathrm{F} 1} \\
& {[z]=[z] \cap[x] \cdot[a]} \\
& \text { F2 } \\
& \text { \%backward propagation } \\
& {[x]=[x] \cap[z] /[a]} \\
& \text { B3 } \\
& {[a]=[a] \cap[z] /[x]} \\
& \text { B4 } \\
& {[y]=[y] \cap\left[\cos ^{-1}\right]([a]) \mathrm{B} 5}
\end{aligned}
$$

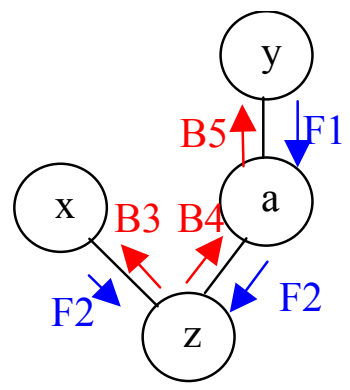

Please, notice that the order of the constraints is important. In the considered example, this order is optimal as shown by the graph. For more details, please see [4].

Suppose that $x \in[x]=[-1 ; 2], y \in[y]=[0 ; \pi / 2], z \in[z]=[1 ; 3]$. In this case, the FPB contractor gives the following results:

$$
\begin{aligned}
& \mathrm{F} 1:[a]=[-\infty,+\infty] \cap[\cos ]([0, \pi / 3])=[0.5,1] \\
& \text { F2: }[z]=[1,3] \cap[-1,2] \cdot[0.5,1]=[1,2] \\
& \text { B3: }[x]=[-1,2] \cap[1,2] /[0.5,1]=[1,2] \\
& \text { B4: }[a]=[0.5,1] \cap[1,2] /[1,2]=[0.5,1] \\
& \text { B5: }[y]=[0, \pi / 2] \cap\left[\cos ^{-1}\right]([0.5,1])=[0, \pi / 3]
\end{aligned}
$$

When there are several redundant constraints, the graph corresponding to the elementary constraints contains cycles, in general. In that case, the FBP contractor may not reach a locally consistence. A solution is then to apply the algorithm of Waltz whose principle is to repeat the propagation until the intervals do not contract any more [3].

\section{DATA FUSION USING THE FBP CONTRACTOR}

\section{A. Global architecture of the multi-sensor fusion}

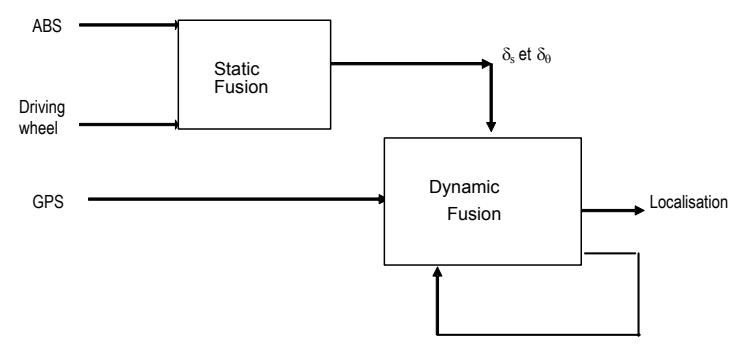

Fig. 2. Localizer architecture.

For a better clearness, let consider two levels of fusion as shown on figure 2 . The elementary rotation and displacement $\delta_{\theta, k}$ et $\delta_{s, k}$ are given by a static fusion which uses the measurements of the ABS sensors of the 4 wheels and the driving wheel encoder. The redundancy of the information provided by these sensors should decrease in a significant way, the pessimism of the initial box. The result is the input of the dynamic module which computes the vehicle location.

\section{B. Models used}

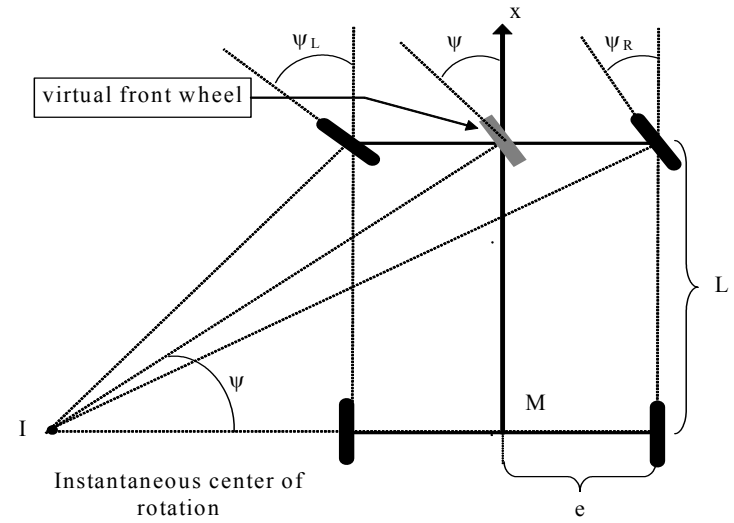

Fig. 3. The Ackerman model of a car in a turning maneuver.

For the static fusion stage, we consider an Ackerman model (fig (3), see [9]). It provides the non linear system:

$$
\begin{cases}\tan (\psi) & =L \cdot \frac{\delta_{\theta}}{\delta_{S}} \\ \delta_{R L} & =\delta_{S}-e \cdot \delta_{\theta} \\ \delta_{R R} & =\delta_{S}+e \cdot \delta_{\theta} \\ \delta_{F L} \cdot \cos \left(\psi_{L}\right) & =\delta_{S}-e \cdot \delta_{\theta} \\ \delta_{F R} \cdot \cos \left(\psi_{R}\right) & =\delta_{S}+e \cdot \delta_{\theta} \\ \tan \left(\psi_{L}\right) & =\frac{L \cdot \tan (\psi)}{L-e \cdot \tan (\psi)} \\ \tan \left(\psi_{R}\right) & =\frac{L \cdot \tan (\psi)}{L+e \cdot \tan (\psi)}\end{cases}
$$

Where:

- $\delta_{R L}, \delta_{R R}, \delta_{F L}, \delta_{F R}, \psi$ denote the measured variables (the distances travelled between two samples by the different wheels and the angle of a virtual wheel, measured by the driving wheel encoder);

- $\delta_{s}, \delta_{\theta}, \psi_{R}, \psi_{L}$ are the estimated variables (the elementary distance, the angle between two samples and the angles of the two front wheels);

- $\quad L$ and $e$ are the vehicle parameters (the distance between the axles and the wheel base).

In the dynamic fusion stage, we consider an odometric model. The mobile vehicle pose $X_{k}$, at time instant $k$, is calculated thanks to $X_{k-I}$ and to the output of the static fusion process by: 


$$
\left\{\begin{array}{l}
x_{k+1}=x_{k}+\delta_{s, k} \cdot \cos \left(\theta_{k}+\delta_{\theta, k} / 2\right) \\
y_{k+1}=y_{k}+\delta_{s, k} \cdot \sin \left(\theta_{k}+\delta_{\theta, k} / 2\right) \\
\theta_{k+1}=\theta_{k}+\delta_{\theta, k}
\end{array}\right.
$$

where $x_{k}$ and $y_{k}$ represent the vehicle position, at time $t_{k}$, in the reference frame and $\theta_{k}$ the heading angle.

\section{Static fusion}

The aim of the static fusion stage is to compute guaranteed intervals for $\delta_{\theta, k}$ et $\delta_{s, k}$. At every moment $\mathrm{t}_{\mathrm{k}}$, let consider the CSP $H_{\mathrm{k}}:(\mathrm{F}(\mathrm{x})=0 / \mathrm{x} \in[\mathrm{x}])$, where:

- $\quad x=\left(\delta s, \delta_{R L}, \delta_{R R}, \delta_{F L}, \delta_{F R}, \delta_{\theta}, \psi, \psi_{R}, \psi_{L}, L, e\right)$

- $\quad$ F represents the system of equations (5)

- The 4 ABS provide $\left[\delta_{R L}\right],\left[\delta_{R R}\right],\left[\delta_{F L}\right],\left[\delta_{F R}\right]$. In order to determine borders for the intervals, we suppose that the covered distance error between two instants $t_{k-1}$ and $t_{k}$, is less than the covered distance corresponding to one step of the ABS sensor (denoted $\delta_{A B S}$ ), with the hypothesis that the vehicle rolls without slipping.

$$
[\delta]=\left[\delta_{\text {mes }}-\delta_{A B S}, \delta_{\text {mes }}+\delta_{A B S}\right]
$$

- The error of the angle $\psi$ is linked to the error of the driving wheel encoder. Thanks to specific tests, we estimated that the maximum of this error is $\psi_{D W E}=0.5 \mathrm{deg}$.

$$
[\psi]=\left[\psi_{m e s}-\psi_{D W E}, \psi_{m e s}+\psi_{D W E}\right]
$$

- $\left[\delta_{S}\right],\left[\delta_{\theta}\right],\left[\psi_{R}\right],\left[\psi_{L}\right]$ are not measured. They are initialized with unknown value: $[-\infty,+\infty]$

- $\quad[\mathrm{L}],[\mathrm{e}]$ are vehicle parameters. They are roughly known that's why they are treated as unknown quantities in the FBP.

The estimates of $\left[\delta_{\theta, k}\right]$ et $\left[\delta_{s, k}\right]$ are obtained thanks to the FBP contractor (presented in section II.D) applied to the CSP $H_{\mathrm{k}}$. Please notice that between two samples the CSP are different and independent. In addition, an originality of the method is that all the variables of the CSP can be contracted even the measurements and the parameters used in the model. The appendix presents the algorithm we have developed.

\section{Dynamic fusion}

For every moment $k$, let consider all the state equations between the time indexes 0 and $k$. In theory, we have to solve the complete CSP $H:(\mathrm{F}(\mathrm{x})=0 / \mathrm{x} \in[\mathrm{x}])$, where: $x=\left(x_{k}, \ldots, x_{0}, y_{k}, \ldots\right.$,

$$
\left.y_{0}, \theta_{k}, \ldots, \theta_{0}, \delta_{S, k}, \ldots, \delta_{S, 0}, \delta_{\theta, k}, \ldots, \delta_{\theta, 0}\right)
$$

- $\left[\delta_{S, i}\right]$ et $\left[\delta_{\theta, i}\right]$ are provided by the static fusion stage
- The GPS measurement ( $\left.\mathrm{x}_{\mathrm{gps}, \mathrm{mes}}, \mathrm{y}_{\mathrm{gps}, \mathrm{mes}}\right)$ is used to initialize the intervals $\left[\mathrm{x}_{\mathrm{k}}\right]$ and $\left[\mathrm{y}_{\mathrm{k}}\right]$. The longitude/latitude estimated point is converted to his Cartesian coordinate, in a local frame (see Fig. (6)). The GPS bounded error measurement is obtained as it follows. The GST NMEA sentence is used to characterize the error bound which is taken such as 3 times the estimated standard deviation $\hat{\sigma}$ (computed in real time by the GPS receiver).

$$
\begin{aligned}
& {\left[x_{g p s}\right]=\left[x_{g p s, m e s}-3 \hat{\sigma}_{x}, x_{g p s, m e s}+3 \hat{\sigma}_{x}\right]} \\
& {\left[y_{g p s}\right]=\left[y_{g p s, m e s}-3 \hat{\sigma}_{y}, y_{g p s, m e s}+3 \hat{\sigma}_{y}\right]}
\end{aligned}
$$

- The heading angle $\left[\theta_{\mathrm{i}}\right]=[-\infty,+\infty]$ is not measured

- $\quad$ F represents the system of odometric model (6).

This CSP gives a general solution the non linear state observation [5]. For a real time implementation of this method, it is unrealistic to consider all the equations from $t_{0}$ to $t_{k}$. Therefore, we consider a limited horizon ' $h$ '. Typically, we have considered a horizon equal to 4 (i.e. 4 samples) which induces 27 primitives constraints. Please notice that the inputs $\delta_{S, i}, \delta_{\theta, i}$, are contracted a second time in $H$.
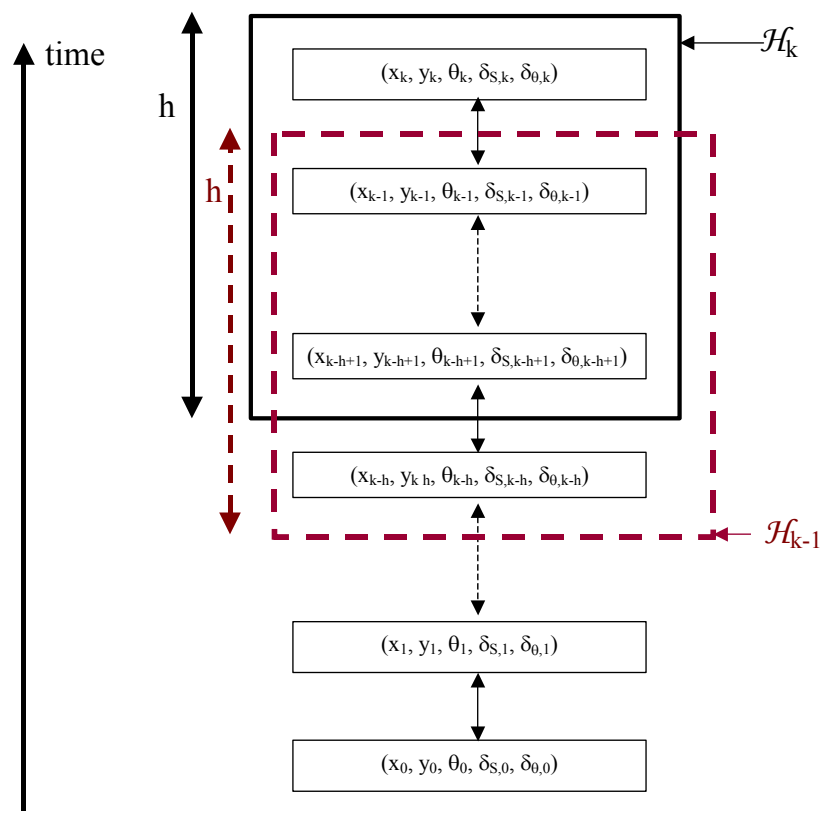

Fig. 4. Graphical interpretation of the horizon limited CSP $\mathcal{H}$.

\section{EXPERIMENTAL RESULTS}

Several tests have been carried out with our experimental car (Fig. 5). The data of the four ABS, the driving wheel encoder and the Trimble Ag132 differential GPS receiver (working with Omnistar differential corrections) have been synchronized thanks to the Pulse Per Second output of the GPS 
receiver The sampling rate was $5 \mathrm{~Hz}$. The data of an optical gyro KVH has been also collected for comparison purposes.

Figure 6 shows the top view of an experiment. In the continuation, we will limit our study to the part framed in black. It contains two roundabouts, a straight line and a turn.

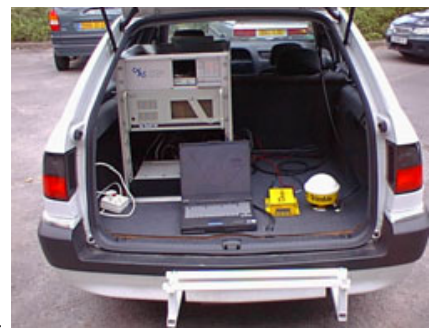

Fig. 5. View of the experimental car with the Ag132 GPS receiver.

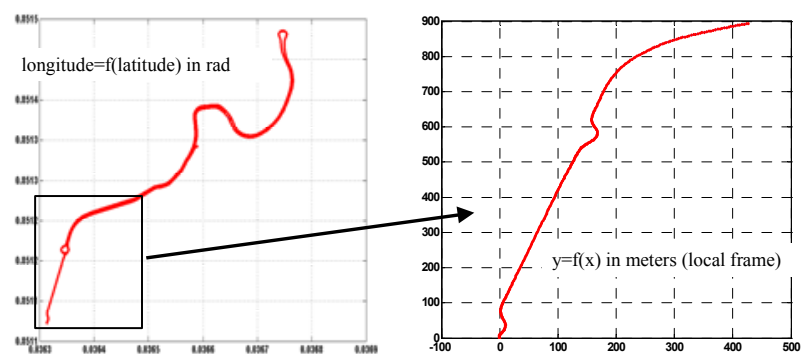

6. Overview of the trial.

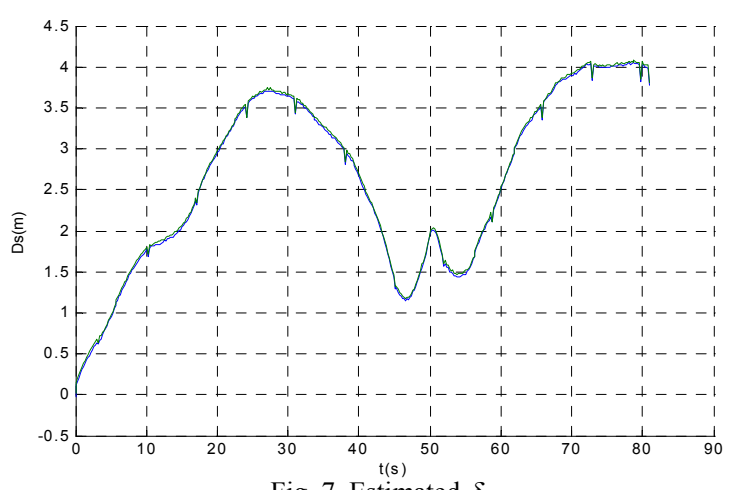

Fig. 7. Estimated $\delta_{s}$.

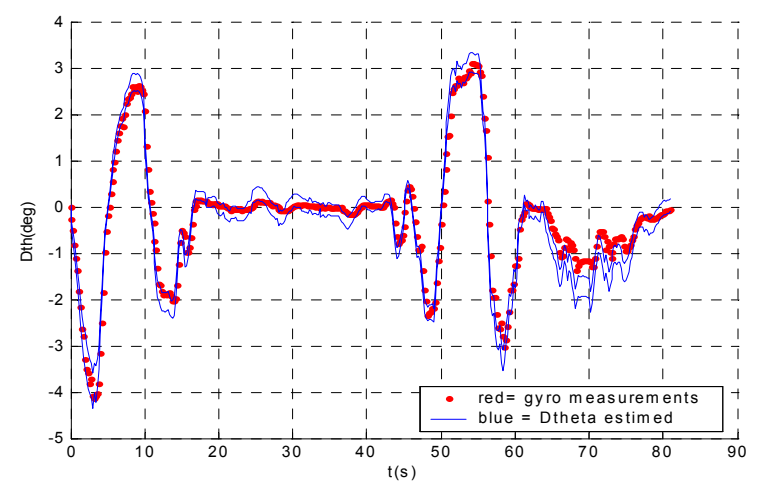

Fig. 8. Estimated $\delta_{\theta}$. The data of the KVH gyro is dotted.
The results of the static fusion are plotted on figures 7 and 8 . Each estimate represents a guaranteed elementary displacement or rotation during $200 \mathrm{~ms}$. The irregularities of figure 8 are due to the fact that the acquisition process is not perfectly periodic. Nevertheless, the method we have used is insensible to this phenomenon since the odometric model does not require a strictly periodic sampling.

The estimated elementary rotation is very good (+/- $0.2 \mathrm{deg}$.) and very close to the measurement provided by a fiber optic gyro (Fig. 8).

Figure 9 indicates that the ensemblist fusion of all the sensors reduces significantly the uncertainty while ensuring guaranteed estimates. Moreover, one can remark that the FBP provides a dead reckoning estimate of the pose of the car when the GPS signal is blocked. This estimate is also guaranteed. On Fig. 9, the duration of the GPS failure is 10 seconds.

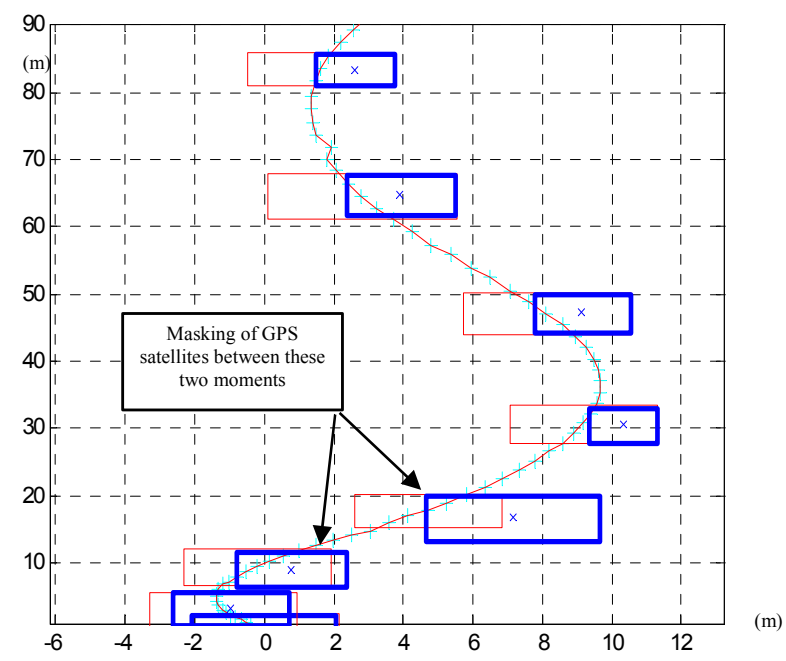

Fig. 9. Estimated location boxes in meters (DGPS in thin and FPB in bold).

Finally, the FBP contractor is able to reconstruct a non directly observed quantity, like the heading angle (see Fig.10). Please keep in mind that this estimate is the result of a guaranteed non linear state observation.

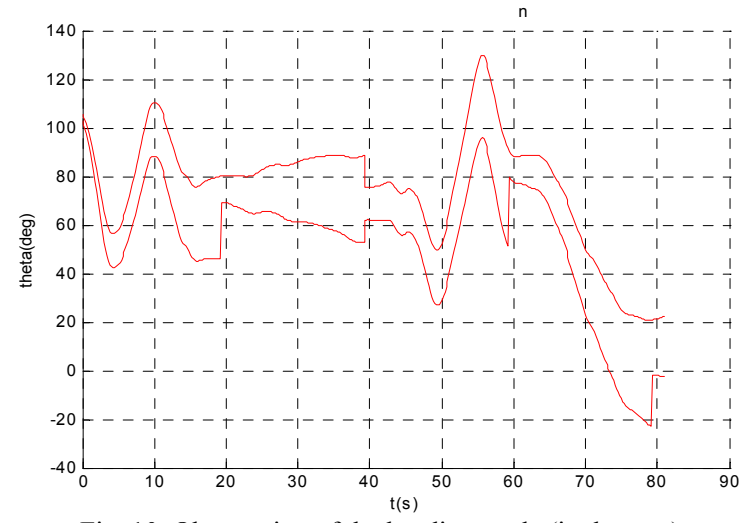

Fig. 10. Observation of the heading angle (in degrees). 
For purposes, the static fusion stage treats one minute of data in roughly $30 \mathrm{~s}$ and the dynamic fusion stage in $15 \mathrm{~s}$, with a 1.8 GHz Pentium 4 (Matlab implementation).

\section{CONCLUSION}

This paper has presented a new dynamic localization technique based on constraints propagation on real intervals. This approach guarantees that the real pose of the car is included in the estimated boxes.

In the problem treated here, there is a high redundancy in data and equations since we have used the 4 ABS encoders, the driving wheel encoder and the measurements of a DGPS receiver. All this information is available in modern cars. Thanks to this redundancy, contractors alone can be used (i.e. without bisection) since the consistence of all the data produces rather precise estimates (i.e. not too pessimistic) as shown by the experimental results. The contractor presented is based on Forward and Backward Propagation (FBP) in addition with the use of the Waltz algorithm for the cycles in the constraints graph, in order to optimise the contraction. As shown, this method is well adapted to a real time context.

The major implementation problem of such an approach is to determine correctly the bounds of the noises. Indeed, if these bounds are underestimated, the contractor can say that there is no solution. On the contrary, if the bounds are overestimated, the estimated boxes can be very large (the estimates are then very pessimistic). A methodological way to treat the tuning of these parameters represents the main perspective of this research.

\section{REFERENCES}

[1] D. Meizel, O. Lévêque, L. Jaulin and E. Walter. "Initial Localization by Set Inversion". IEEE transactions on. on robotics and Automation. Volume 18, Number 6, pages 966-971. 2002.

[2] D. Bouvet and G. Garcia. "Guaranteed 3-D mobile robot localization using an odometer, an automatic theodolite and indistinguishable landmarks". ICRA 01.pp 3612-3617.

[3] D. Waltz. "Generating semantic descriptions from drawings of scenes with shadows", The psychology of computer Vision, New York, NY pp. 19-91(1975)

[4] F. Benhamou, F. Goualard, L. Granvilliers, and J.F. Puget. "Revising hull and box consistency", Proceedings of the Intervational Conference on Logic Programming, Las Cruces, NM, pp.230-244. 1999.

[5] L. Jaulin. "Nonlinear bounded-error state estimation of continuoustime systems". Automatica, 38, 1079-1082. 2002.

[6] L. Jaulin., M. Kieffer, O. Didrit and E. Walter. “Applied Interval Analysis", Springer-Verlag, (2001)

[7] M. Kieffer, L. Jaulin and E. Walter. "Guaranteed recursive nonlinear state estimation using interval Analysis", International journal of adaptative control and signal processing, 16:193-218, 2002.
[8] P. Bouron, D. Meizel, Ph. Bonnifait. "Set-Membership Non-Linear Observers with Application to Vehicle Localisation". 6th European Control Conference. Porto. Sept 2001.

[9] Ph. Bonnifait, P. Bouron, P. Crubillé, D. Meizel. "Data Fusion of Four ABS Sensors and GPS for an Enhanced Localization of Car-like Vehicles", IEEE International Conference on Robotics and Automation (ICRA 01). Séoul. May 2001. pp. 1597-1602.

[10] R. E. Moore, "Interval Analysis", Prentice-Hall, Englewood Cliffs, NJ, 1966.

[11] S.A Malan, M. Milanese, M. Taragna, And J Garloff. " $\mathrm{B}^{3}$ Algorithm for Robust Performance Analysis in Presence of Mixed Parametric and Dynamic Perturbations", Proceedings of the $31^{\text {st }}$ IEEE Conference on Decision and control, Tucson, 1992. AZ, pp.128-133

[12] S. Sukkarieh, E. M. Nebot, HF. Durrant-Whyte. "Achieving Integrity in an INS/GPS Navigation Loop for Autonomous Land Vehicle Applications". IEEE International Conference on Robotics and Automation. (ICRA98).Leuven, Belgium May 1998. pp 3437-3442

\section{APPENDIX: FBP CONTRACTOR FOR THE STATIC FUSION}

First, let introduce auxiliary variables $a_{i}$ for $i \in[1,8]$ to get primitive constraints. Then, we apply the FBP algorithm: While it contracts "enough", repeat

\begin{tabular}{|c|c|}
\hline Forward propagation & Backward propagation \\
\hline$\left[\mathrm{a}_{1, \mathrm{i}}\right]=\left[\mathrm{a}_{1, \mathrm{i}}\right] \cap[\tan ]\left(\left[\psi_{\mathrm{i}}\right]\right) \cdot\left[\mathrm{L}_{\mathrm{i}}\right]$ & {$\left[\mathrm{a}_{8, \mathrm{i}}\right]=\left[\mathrm{a}_{8, \mathrm{i}}\right] \cap\left[\delta_{\theta, \mathrm{i}} \mathrm{i}\right] /\left[\delta_{\mathrm{S}, \mathrm{i}}\right]$} \\
\hline$\left[\mathrm{a}_{2, \mathrm{i}}\right]=\left[\mathrm{a}_{2, \mathrm{i}}\right] \cap[\tan ]\left(\left[\psi_{\mathrm{i}}\right]\right) \cdot\left[\mathrm{E}_{\mathrm{i}}\right]$ & {$\left[\psi_{\mathrm{i}}\right]=\left[\psi_{\mathrm{i}}\right] \cap\left[\tan ^{-1}\right]\left(\left[\mathrm{L}_{\mathrm{i}}\right] \cdot\left[\mathrm{a}_{8, \mathrm{i}}\right]\right)$} \\
\hline$\left[\mathrm{a}_{3, \mathrm{i}}\right]=\left[\mathrm{a}_{3, \mathrm{i}}\right] \cap\left[\mathrm{L}_{\mathrm{i}}\right]-\left[\mathrm{a}_{2, \mathrm{i}}\right]$ & {$\left[\mathrm{L}_{\mathrm{i}}\right]=\left[\mathrm{L}_{\mathrm{i}}\right] \cap[\tan ]\left(\left[\psi_{\mathrm{i}}\right]\right) /\left[\mathrm{a}_{8, \mathrm{i}}\right]$} \\
\hline$\left[\psi_{\mathrm{L}, \mathrm{i}}\right]=\left[\psi_{\mathrm{L}, \mathrm{i}}\right] \cap\left[\tan ^{-1}\right]\left(\left[\mathrm{a}_{1, \mathrm{i}}\right] /\left[\mathrm{a}_{3, \mathrm{i}}\right]\right)$ & {$\left[\mathrm{a}_{7, \mathrm{i}}\right]=\left[\mathrm{a}_{7, \mathrm{i}}\right] \cap\left[\delta_{\mathrm{RR}, \mathrm{i}}\right]-\left[\delta_{\mathrm{S}, \mathrm{i}}\right]$} \\
\hline$\left[\mathrm{a}_{4, \mathrm{i}}\right]=\left[\mathrm{a}_{4, \mathrm{i}}\right] \cap\left[\mathrm{L}_{\mathrm{i}}\right]+\left[\mathrm{a}_{2, \mathrm{i}}\right]$ & {$\left[\mathrm{a}_{7, \mathrm{i}}\right]=\left[\mathrm{a}_{7, \mathrm{i}}\right] \cap\left[\delta_{\mathrm{S}, \mathrm{i}}\right]-\left[\delta_{\mathrm{RL}, \mathrm{i}}\right]$} \\
\hline$\left[\psi_{\mathrm{R}, \mathrm{i}}\right]=\left[\psi_{\mathrm{R}, \mathrm{i}}\right] \cap\left[\tan ^{-1}\right]\left(\left[\mathrm{a}_{1, \mathrm{i}}\right] /\left[\mathrm{a}_{4, \mathrm{i}}\right]\right)$ & {$\left[\mathrm{a}_{7, \mathrm{i}}\right]=\left[\mathrm{a}_{7, \mathrm{i}}\right] \cap\left[\mathrm{a}_{5, \mathrm{i}}\right]-\left[\delta_{\mathrm{S}, \mathrm{i}}\right]$} \\
\hline$\left[\mathrm{a}_{5, \mathrm{i}}\right]=\left[\mathrm{a}_{5, \mathrm{i}}\right] \cap\left[\delta_{\mathrm{FR}, \mathrm{i}}\right] \cdot[\cos ]\left(\left[\psi_{\mathrm{R}, \mathrm{i}}\right]\right)$ & {$\left[\mathrm{a}_{7, \mathrm{i}}\right]=\left[\mathrm{a}_{7, \mathrm{i}}\right] \cap\left[\delta_{\mathrm{S}, \mathrm{i}}\right]-\left[\mathrm{a}_{6, \mathrm{i}}\right]$} \\
\hline$\left[\mathrm{a}_{6, \mathrm{i}}\right]=\left[\mathrm{a}_{6, \mathrm{i}}\right] \cap\left[\delta_{\mathrm{FL}, \mathrm{i}}\right] \cdot[\cos ]\left(\left[\psi_{\mathrm{L}, \mathrm{i}}\right]\right)$ & {$\left[\delta_{R L, i}\right]=\left[\delta_{R L, i}\right] \cap\left[\delta_{S, i}\right]-\left[a_{7, i}\right]$} \\
\hline$\left[\mathrm{a}_{7, \mathrm{i}}\right]=\left[\mathrm{a}_{7, \mathrm{i}}\right] \cap\left[\delta_{\theta, \mathrm{i}}\right] \cdot\left[\mathrm{E}_{\mathrm{i}}\right]$ & {$\left[\delta_{R R, i}\right]=\left[\delta_{R R, i}\right] \cap\left[\delta_{S, i}\right]+\left[a_{7, i}\right]$} \\
\hline$\left[\delta_{\mathrm{S}, \mathrm{i}}\right]=\left[\delta_{\mathrm{S}, \mathrm{i}}\right] \cap\left[\mathrm{a}_{5, \mathrm{i}}\right]-\left[\mathrm{a}_{7, \mathrm{i}}\right]$ & {$\left[\mathrm{E}_{\mathrm{i}}\right]=\left[\mathrm{E}_{\mathrm{i}}\right] \cap\left[\mathrm{a}_{7, \mathrm{i}}\right] /\left[\delta_{\theta, \mathrm{i}}\right]$} \\
\hline$\left[\delta_{\mathrm{S}, \mathrm{i}}\right]=\left[\delta_{\mathrm{S}, \mathrm{i}}\right] \cap\left[\mathrm{a}_{6, \mathrm{i}}\right]+\left[\mathrm{a}_{7, \mathrm{i}}\right]$ & {$\left[\delta_{\theta, \mathrm{i}}\right]=\left[\delta_{\theta, \mathrm{i}}\right] \cap\left[\mathrm{a}_{7, \mathrm{i}}\right] /\left[\mathrm{E}_{\mathrm{i}}\right]$} \\
\hline$\left[\delta_{\mathrm{S}, \mathrm{i}}\right]=\left[\delta_{\mathrm{S}, \mathrm{i}}\right] \cap\left[\delta_{\mathrm{RR}, \mathrm{i}}\right]-\left[\mathrm{a}_{7, \mathrm{i}}\right]$ & {$\left[\mathrm{a}_{6, \mathrm{i}}\right]=\left[\mathrm{a}_{6, \mathrm{i}}\right] \cap\left[\delta_{\mathrm{S}, \mathrm{i}}\right]-\left[\mathrm{a}_{7, \mathrm{i}}\right]$} \\
\hline$\left[\delta_{\mathrm{S}, \mathrm{i}}\right]=\left[\delta_{\mathrm{S}, \mathrm{i}}\right] \cap\left[\delta_{\mathrm{RL}, \mathrm{i}}\right]+\left[\mathrm{a}_{7, \mathrm{i}}\right]$ & {$\left[\mathrm{a}_{5, \mathrm{i}}\right]=\left[\mathrm{a}_{5, \mathrm{i}}\right] \cap\left[\delta_{\mathrm{S}, \mathrm{i}}\right]+\left[\mathrm{a}_{7, \mathrm{i}}\right]$} \\
\hline$\left[\mathrm{a}_{8, \mathrm{i}}\right]=\left[\mathrm{a}_{8, \mathrm{i}}\right] \cap[\tan ]\left(\left[\psi_{\mathrm{i}}\right]\right) /\left[\mathrm{L}_{\mathrm{i}}\right]$ & {$\left[\delta_{\mathrm{FL}, \mathrm{i}}\right]=\left[\delta_{\mathrm{FL}, \mathrm{i}}\right] \cap\left[\mathrm{a}_{6, \mathrm{i}}\right] /[\cos ]\left(\left[\psi_{\mathrm{L}, \mathrm{i}}\right]\right)$} \\
\hline$\left[\delta_{\theta, \mathrm{i}}\right]=\left[\delta_{\theta, \mathrm{i}}\right] \cap\left[\mathrm{a}_{8, \mathrm{i}}\right] \cdot\left[\delta_{\mathrm{S}, \mathrm{i}}\right]$ & {$\left[\psi_{\mathrm{L}, \mathrm{i}}\right]=\left[\psi_{\mathrm{L}, \mathrm{i}}\right] \cap\left[\cos ^{-1}\right]\left(\left[\mathrm{a}_{6, \mathrm{i}}\right] /\left[\delta_{\mathrm{FL}, \mathrm{i}}\right]\right)$} \\
\hline$\left[\delta_{\mathrm{S}, \mathrm{i}}\right]=\left[\delta_{\mathrm{S}, \mathrm{i}}\right] \cap\left[\delta_{\theta, \mathrm{i}}\right] /\left[\mathrm{a}_{8, \mathrm{i}}\right]$ & {$\left[\delta_{\mathrm{FR}, \mathrm{i}}\right]=\left[\delta_{\mathrm{FR}, \mathrm{i}}\right] \cap\left[\mathrm{a}_{5, \mathrm{i}} \mathrm{i} /[\cos ]\left(\left[\psi_{\mathrm{R}, \mathrm{i}}\right]\right)\right.$} \\
\hline & {$\left[\psi_{\mathrm{R}, \mathrm{i}}\right]=\left[\psi_{\mathrm{R}, \mathrm{i}}\right] \cap\left[\cos ^{-1}\right]\left(\mathrm{a}_{5, \mathrm{i}} / \delta_{\mathrm{FR}, \mathrm{i}}\right)$} \\
\hline & {$\left[\mathrm{a}_{1, \mathrm{i}}\right]=\left[\mathrm{a}_{1, \mathrm{i}}\right] \cap\left[\mathrm{a}_{4, \mathrm{i}}\right] \cdot[\tan ]\left(\left[\psi_{\mathrm{R}, \mathrm{i}}\right]\right)$} \\
\hline & {$\left[\mathrm{a}_{1, \mathrm{i}}\right]=\left[\mathrm{a}_{1, \mathrm{i}}\right] \cap\left[\mathrm{a}_{3, \mathrm{i}}\right] \cdot[\tan ]\left(\left[\psi_{\mathrm{L}, \mathrm{i}}\right]\right)$} \\
\hline & {$\left[\mathrm{a}_{4, \mathrm{i}}\right]=\left[\mathrm{a}_{4, \mathrm{i}}\right] \cap\left[\mathrm{a}_{1, \mathrm{i}}\right] /[\tan ]\left(\left[\psi_{\mathrm{R}, \mathrm{i}}\right]\right)$} \\
\hline & {$\left[\mathrm{a}_{3, \mathrm{i}}\right]=\left[\mathrm{a}_{3, \mathrm{i}}\right] \cap\left[\mathrm{a}_{1, \mathrm{i}}\right] /[\tan ]\left(\left[\psi_{\mathrm{L}, \mathrm{i}}\right]\right)$} \\
\hline & {$\left[\mathrm{L}_{\mathrm{i}}\right]=\left[\mathrm{L}_{\mathrm{i}}\right] \cap\left[\mathrm{a}_{3, \mathrm{i}}\right]+\left[\mathrm{a}_{2, \mathrm{i}}\right]$} \\
\hline & {$\left[\mathrm{L}_{\mathrm{i}}\right]=\left[\mathrm{L}_{\mathrm{i}}\right] \cap\left[\mathrm{a}_{4, \mathrm{i}}\right]-\left[\mathrm{a}_{2, \mathrm{i}}\right]$} \\
\hline & {$\left[\mathrm{a}_{2, \mathrm{i}}\right]=\left[\mathrm{a}_{2, \mathrm{i}}\right] \cap\left[\mathrm{a}_{4, \mathrm{i}}\right]-\left[\mathrm{L}_{\mathrm{i}}\right]$} \\
\hline & {$\left[\mathrm{a}_{2, \mathrm{i}}\right]=\left[\mathrm{a}_{2, \mathrm{i}}\right] \cap\left[\mathrm{L}_{\mathrm{i}}\right]-\left[\mathrm{a}_{3, \mathrm{i}}\right]$} \\
\hline & {$\left[\mathrm{E}_{\mathrm{i}}\right]=\left[\mathrm{E}_{\mathrm{i}}\right] \cap\left[\mathrm{a}_{2, \mathrm{i}}\right] /[\tan ]\left(\left[\psi_{\mathrm{i}}\right]\right)$} \\
\hline & {$\left[\psi_{\mathrm{i}}\right]=\left[\psi_{\mathrm{i}}\right] \cap \tan ^{-1}\left(\left[\mathrm{a}_{2, \mathrm{i}}\right] /\left[\mathrm{E}_{\mathrm{i}}\right]\right)$} \\
\hline & {$\left[\mathrm{L}_{\mathrm{i}}\right]=\left[\mathrm{L}_{\mathrm{i}}\right] \cap\left[\mathrm{a}_{1, \mathrm{i}}\right] /[\tan ]\left(\left[\psi_{\mathrm{i}}\right]\right)$} \\
\hline & {$\left[\psi_{\mathrm{i}}\right]=\left[\psi_{\mathrm{i}}\right] \cap\left[\tan ^{-1}\right]\left(\left[\mathrm{a}_{1, \mathrm{i}}\right] /\left[\mathrm{L}_{\mathrm{i}}\right]\right)$} \\
\hline
\end{tabular}

